\title{
Stable and Low-Threshold Optical Gain in CdSe/CdS Quantum Dots: An All-Colloidal Frequency Up-Converted \\ Laser
}

\author{
Burak Guzelturk, Yusuf Kelestemur, Kivanc Gungor, Aydan Yeltik, Mehmet Zafer Akgul, \\ Yue Wang, Rui Chen, Cuong Dang,* Handong Sun,* and Hilmi Volkan Demir*
}

Current semiconductor lasers are experiencing technological challenges to access the green and yellow regions of the spectrum. ${ }^{[1]}$ With quantum-confinement effects, colloidal quantum dots (QDs) are artificial atoms whose optical properties can be widely tuned via shape, size, and composition during a colloidal-synthesis process. ${ }^{[2]}$ QD-based optical gain media can be precisely controlled to make a laser at any wavelength in the entire visible spectrum, ${ }^{[3-7]}$ and further in the near-infrared region. ${ }^{\left[{ }^{[]}\right.}$Besides the advantages of color tunability and chemical processability, QDs promise temperature-insensitive optical gain because of well-separated energy levels and the atomic-like states. ${ }^{[9,10]}$ To date, optical gain has been shown with various colloidal core/shell QDs. ${ }^{[3,11-14]}$ However, the small volume of nanometer-sized QDs substantially increases the non-radiative multiexciton Auger recombination (AR), creating a significantly large non-radiative channel for depleting exciton energy into heat. ${ }^{[15,16]}$ The single exciton gain in type-I QDs is an advantageous mechanism to eliminate the multiexciton AR process and therefore to enable low-threshold QD gain media. ${ }^{[3,7]}$ Nevertheless, the gain performance would still be limited by the extremely fast AR rate when working at higher pump intensities. Reducing the wave-function overlap of the electron and hole was demonstrated to reduce the AR rate through

\footnotetext{
B. Guzelturk, Y. Kelestemur, K. Gungor, A. Yeltik, M. Z. Akgul, Prof. H. V. Demir

Department of Electrical and Electronics Engineering

Department of Physics, and UNAM-National

Nanotechnology Research Center

Institute of Materials Science and Nanotechnology

Bilkent University

TR-06800, Bilkent, Ankara, Turkey

E-mail:volkan@bilkent.edu.tr; volkan@stanfordalumni.org

Y. Wang, Dr. R. Chen, Prof. H. Sun, Prof. H. V. Demir

Division of Physics and Applied Physics

Centre for Disruptive Photonic Technologies (CDPT)

School of Physical and Mathematical Sciences

Nanyang Technological University

21 Nanyang Link, Singapore 637371, Singapore

E-mail: hdsun@ntu.edu.sg; hvdemir@ntu.edu.sg

Prof. C. Dang, Prof. H. V. Demir

Luminous! Center of Excellence for Semiconductor

Lighting and Displays, TPI-The Photonics Institute

School of Electrical and Electronic Engineering

School of Physical and Mathematical Sciences

Nanyang Technological University

Nanyang Avenue, Singapore 639798, Singapore

E-mail: hcdang@ntu.edu.sg

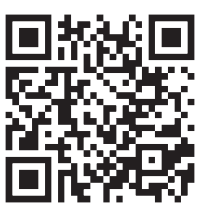

engineering the energy levels and the size of the core and the shell. ${ }^{[17,18]}$ This led to a lowered gain threshold, ${ }^{[19]}$ yet the reduction of the electron-hole wave-function overlap in these type-II or quasi type-II structures of quantum rods, ${ }^{[17]}$ or giant QDs, ${ }^{[18]}$ dramatically decreases the optical oscillator strength and gain coefficient. In addition, the optical gain coefficient suffers from the low particle density per volume because of the giant size of these particles. ${ }^{[18]}$

In this study, we show very high performance and extremely stable optical gain media using tailored CdSe-core/CdS-shell QDs via both single- and two-photon absorption (1PA and 2PA, respectively) pumping. We synthesized high-quality zinc-blende (ZB) CdSe nanocrystals followed by slow CdS-shell growth at high temperature to improve the crystal quality. Our relatively thin CdS shell (6 monolayers) greatly helps to advantageously combine the small size (i.e., larger number of particles per volume), high photoluminescence (PL) quantum yield and the high performance optical gain. This results in a low threshold of $29 \mu \mathrm{J} \mathrm{cm}^{-2}$ and extremely high stability while preserving the initial stimulated emission intensity even after $1.8 \times 10^{7}$ laser shots under 1PA. The high-performance QD gain medium is further demonstrated in an all-colloidal vertical-cavity surfaceemitting laser (AC-VCSEL) using simple and solution-processed distributed Bragg reflectors (DBRs) of just $96 \%$ reflectivity. With the solution-processed DBRs and the tailored core/ shell QDs, we have achieved an ultralow frequency up-converted lasing threshold of $764 \mu \mathrm{J} \mathrm{cm}^{-2}$ as compared to the colloidal QDs having thresholds on the order of 5-10 $\mathrm{mJ} \mathrm{cm}^{-2[20,21]}$ and nanorods having a threshold of $910 \mu \mathrm{J} \mathrm{cm}^{-2}$. [22] The allsolution-processed QD-laser represents the most cost-effective approach toward high-performance full-color lasers with single material technology.

The CdSe-core/CdS-shell QDs having a 6-monolayer CdSshell were synthesized by a modified recipe in ref. [23] (see Section S1, Supporting Information). High-temperature synthesis with slow crystal growth and small lattice mismatch allows the shell-growth process to be controlled precisely. Figure 1a and its inset show transmission electron microscopy (TEM) images of the highly single-crystalline quality QDs with monodisperse size distribution, resulting in excellent self-assembly on TEM grid. The crystal structure of the QDs was investigated by the X-ray diffraction (XRD) patterns in Figure 1b. While CdSe core QDs exhibit pure ZB crystal structure, CdSe/CdS core/ shell QDs seem to show diffraction peaks from both the ZB and wurtzite $(\mathrm{W})$ phases. The result suggests that these QDs exhibit ZB-W polytypism, where the wurtzite and ZB domains can be retained within each nanocrystal and can be explained

DOI: 10.1002/adma.201500418 

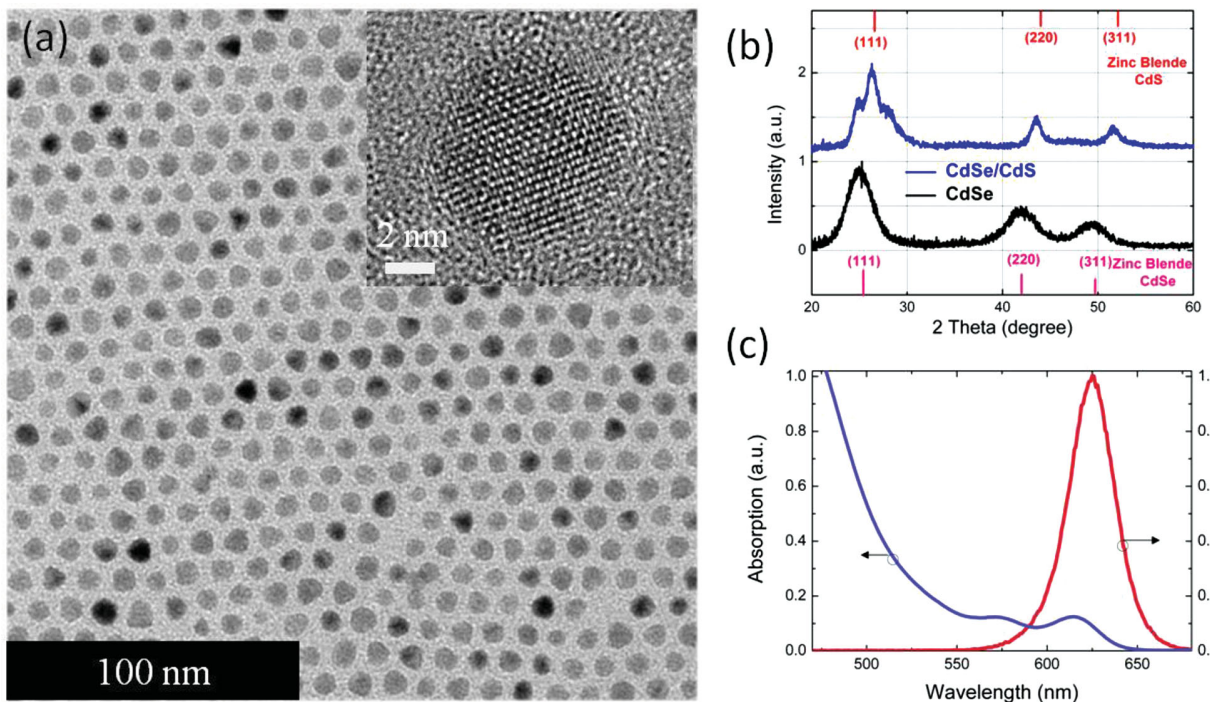

(c)

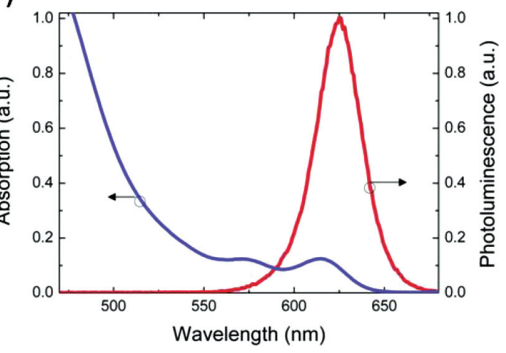

Figure 1. a) TEM image of the CdSe/CdS QDs having CdSe core of $2.2 \mathrm{~nm}$ radius and 6-monolayer CdS shell. The inset shows the high-resolution TEM image of a CdSe/CdS QD with a diameter of $\approx 8 \mathrm{~nm}$. b) XRD patterns of CdSe core (bottom) and CdSe/CdS QDs (top). The CdSe core shows ZB structure with matched XRD pattern to its bulk counterpart. After CdS shell growth, the diffraction peaks are shifted to larger angles and become narrower, indicating the increased crystalline quality and interface-alloying between CdSe and CdS materials. C) PL (red, solid) and absorbance (blue, dotted) of the QDs. Emission line width is less than $30 \mathrm{~nm}$ (FWHM) suggesting the small size distribution.

by the high-temperature CdS shell coating in the presence of primary amines..$^{[2]]}$ The XRD diffractogram in Figure $1 \mathrm{~b}$ also demonstrates the enhanced crystal structure after high-temperature CdS shell growth as observed by the sharper diffraction peaks. The core/shell QD solution shows well-defined excitonic absorption peaks and excellent PL with a quantum yield of as high as $90 \%$ (Figure 1c). In solid films, quantum yield is measured to be as high as $50 \%$ using an integrating sphere.

The dynamics of exciton recombination are studied by timeresolved PL spectroscopy using a streak camera at increasing excitation intensity levels as presented in Figure 2. At the low excitation level $(\langle N\rangle<<0.1)$, slow exponential decay rates are associated with single exciton lifetimes $\left(\tau_{\mathrm{X}}\right)$ which are about $35 \mathrm{~ns}$ for CdSe-core/CdS-shell QDs (Figure 2a) and $\approx 22 \mathrm{~ns}$ for CdSe QDs (Figure 2b) (see Table S1 and S2, Supporting Information for the lifetime components). For the core-only CdSe QDs, there is a small contribution from a fast decay component with a time constant $\tau_{\mathrm{ss}}$ of 1.3-1.8 ns even at extremely low excitation levels. This is attributed to active surface trap states in the core-only CdSe QDs, which deplete excitons via a non-radiative pathway. ${ }^{[25]}$ This also explains the low quantum yields $(<10 \%)$ in these core-only CdSe QDs. Growth of the CdS shell passivates the surface states effectively, resulting in a much higher quantum yield $(\approx 90 \%)$ and the disappearance of such fast decay rate $\left(\tau_{\mathrm{ss}}\right)$. As the pump intensity is increased, we start to observe the buildup of a faster decay component, which is attributed to the decay of the multiexciton $\left(\tau_{\mathrm{xx}}\right)$ where the non-radiative AR has generally a dominant contribution. ${ }^{[10]}$ We calculate $\langle N\rangle=f \times \sigma$ (see Section S3, Supporting Information). In the core-only QDs, for $\langle N\rangle=1.40$ where $\approx 40.5 \%$ of the QD population has multiexcitons (biexciton or higher order excitons) as calculated by the Poisson statistics, a very fast multiexciton lifetime of $\approx 90 \mathrm{ps}$ is observed (see Figure $2 \mathrm{~b}$, inset). This indicates a very fast AR in the core-only QDs. In the case of the core/shell QDs, for $\langle N\rangle=1.23$ where $\approx 35.0 \%$ of the QD population has multiexcitons, a much slower multiexciton lifetime of $\approx 1.25 \mathrm{~ns}$ is observed (Figure 2a). We calculated Auger time constants by $\tau_{\mathrm{AR}}{ }^{-1}=\tau_{\mathrm{XX}}{ }^{-1}-4 \tau_{\mathrm{X}}^{-1}$, where $4 \tau_{\mathrm{X}}{ }^{-1}$ is the radiative recombination rate of the biexciton, and the factor 4 indicates the number of recombination pathways of two electron-hole pairs. These results imply Auger time constants $\tau_{\mathrm{AR}}$ of 91.6 and $1480 \mathrm{ps}$ for the core-only and core/shell QDs, respectively. The Auger time constant (1480 ps) of the CdSe-core/CdS-shell QD structure shows a significant suppression up to an order of magnitude in comparison to that of the CdSe core QDs ${ }^{[15,16,26]}$ and compares well with recent reports using engineered core/ shell QDs. ${ }^{[27,28]}$ This substantial AR suppression is consistent with the recent suppressed blinking observation in similar QDs by ref. [23] and suggests a solution for the fundamentally challenging problem of using QDs for optical amplification.

The nature of the crystal structure should not be accounted for the Auger suppression as our ZB CdSe QDs show a similar Auger time constant to their wurtzite counterparts. The CdS shell is relatively thin; thus, this slightly reduces the overlap of the electron and hole wave functions. This would be one of the reasons for the Auger suppression, as also previously demonstrated in wurtzite QD structures. ${ }^{[19]}$ Such overlap reduction usually lowers the optical oscillator strength, i.e., reduces the radiative recombination rate, and therefore comes with a decreased quantum yield because of the competition with the non-radiative recombination channels. In our core/shell structures, a very high quantum yield is achieved using a thin $\mathrm{CdS}$ shell ( $\approx 6$ monolayers). This suggests that not only an excellent passivation of surface states is achieved but also optical oscillator strength is still high. Another reason for the Auger suppression might be the smooth confinement barrier between the core and shell materials that was also shown to suppresses the blinking in the QDs. ${ }^{[29-31]}$ In QD systems with an abrupt 

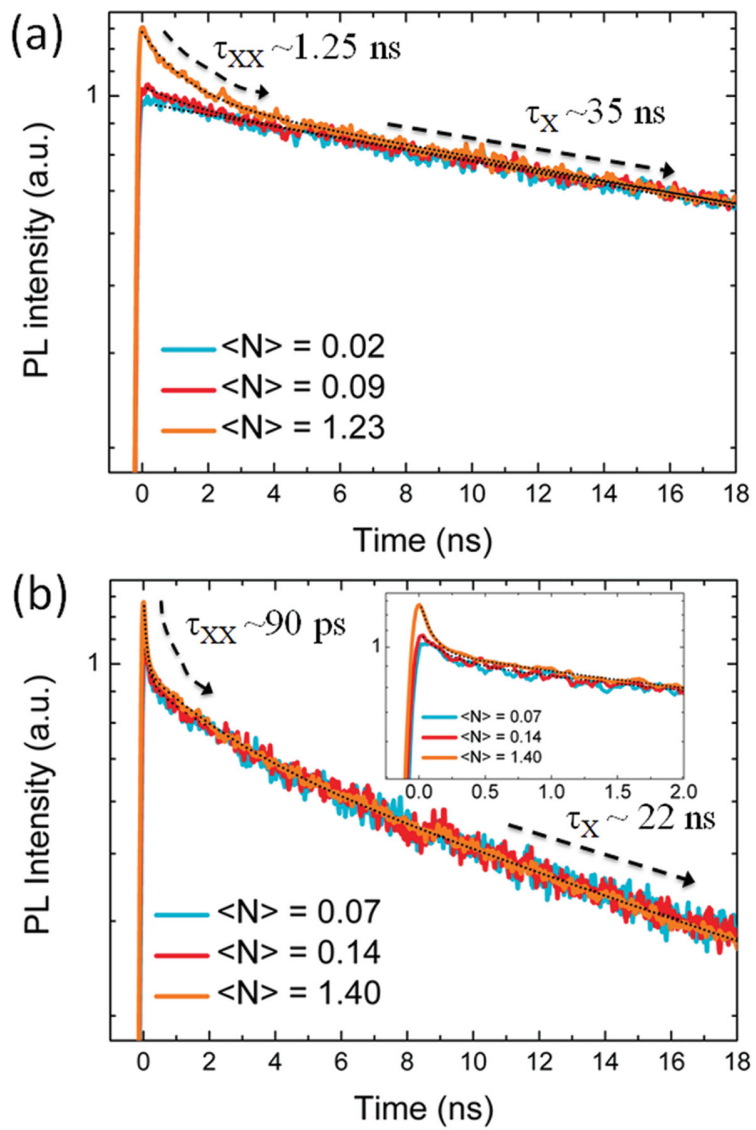

Figure 2. Transient PL intensity measured at the peak emission wavelength at increasing excitation levels for a) $\mathrm{CdSe}-$ core/CdS-shell and b) core-only CdSe QDs. $\tau_{x x}$ is the multiexciton lifetime and $\tau_{x}$ is the single exciton lifetime of the QDs. The average number of excitons per QD $(\langle N\rangle)$ was calculated from the excitation levels (see Section S3, Supporting Information for the calculation). The inset in (b) shows the decay of the CdSe QDs at an early time window.

heterointerface between the core and shell materials, the relaxation of the momentum conservation requirement increases the AR rate significantly. ${ }^{[32]}$ Alloying at the core/shell interface was demonstrated for various heterostructured QD systems including CdSeS/ZnS QDs ${ }^{[33]}$ and wurtzite $\mathrm{CdSe} / \mathrm{CdSeS} / \mathrm{CdS}$ QDs. ${ }^{[18]}$ In our QDs, the CdS shell was grown at an exceptionally high temperature of $310^{\circ} \mathrm{C}$ and at a very slow rate $(6$ monolayers in $3 \mathrm{~h}$ ). Thus, a smooth CdSeS interfaced layer with a thickness of 2-4 monolayers might be created (see Section S5, Supporting Information for the detailed X-ray photoelectron spectroscopy-XPS analysis) due to enhanced interdiffusion of core and shell material and therefore contributing to Auger suppression.

Amplified spontaneous emission (ASE) in the spin-coated core/shell QD films is presented in Figure 3a,b for 1PA and 2PA pumping (see Section S6, Supporting Information for the experimental details), respectively. The onset of stimulated emission in the QD films is clearly shown with the abrupt rising of the output intensity (ASE peak at $\approx 639 \mathrm{~nm}$ ) and the narrowing of the emission spectrum (full width at half maximum $($ FWHM) $\approx 7-9 \mathrm{~nm}$ ) over the threshold pumping
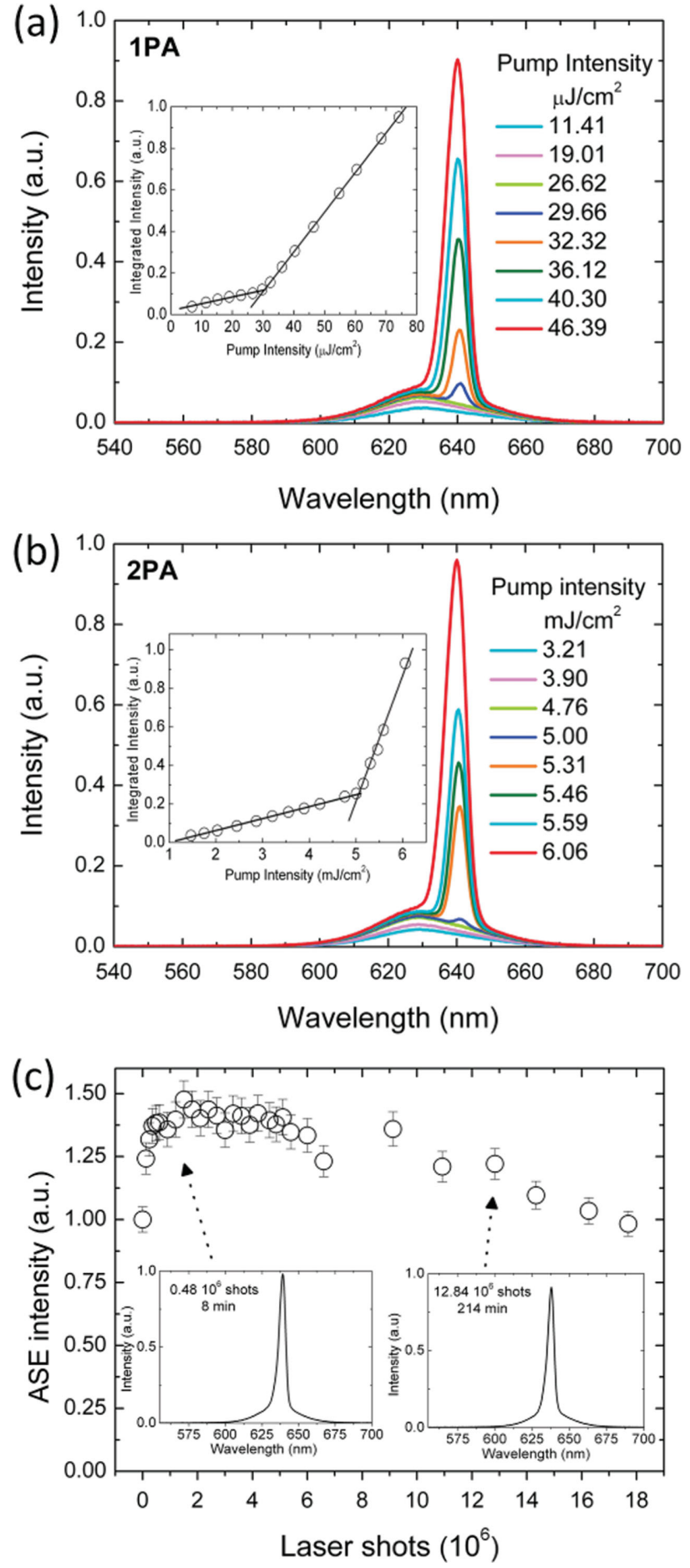

Figure 3. a) The emission spectra of the QD film under 1PA stripe excitation pumping. The inset shows the integrated emission intensity as a function of 1PA pumping energy density. b) 2PA pumped emission spectra of the QDs at various pump intensities. The inset shows the integrated intensity of the emission as a function of 2PA pump intensity. The narrow ASE peak with a FWHM of $5 \mathrm{~nm}$ kicks off at the red-tail of the spontaneous emission spectrum with a FWHM of $\approx 29 \mathrm{~nm}$. c) Laser-shot-dependent ASE intensity under pump laser shots up to $1.8 \times 10^{7}$ indicating the exceptional stability of the QDs. 
intensity. The redshift $(8-10 \mathrm{~nm})$ of the ASE peak in comparison to the PL peak (see Figure $3 \mathrm{a}, \mathrm{b}$ ) is a strong indicator of the biexciton gain mechanism in our type-I QDs due to thin CdS shell growth. ${ }^{[19,34]}$ The slow Auger rate in these core/shell QDs largely helps to reduce the stimulated emission threshold owing to the suppressing the most competitive pathway of multiexciton depletion and therefore enhances biexciton gain. The spin-coated QD films achieved 1PA pumped ASE with a threshold of $29 \mu \mathrm{J} \mathrm{cm}^{-2}$ (Figure 3a, inset), one of the lowest

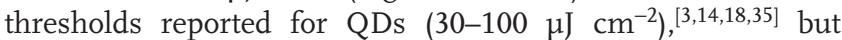
maintained high gain coefficients as high as $120 \mathrm{~cm}^{-1}$ (see Figure S2, Supporting Information). For the case of frequency up-converted, i.e., 2PA, pumping, the threshold for ASE is $\approx 5.02 \mathrm{~mJ} \mathrm{~cm} \mathrm{~cm}^{-2}$ (Figure 3b, inset), the lowest value among redemitting spherical QDs as compared with previous reports. ${ }^{[21,36]}$ On the other hand, our threshold is larger than the threshold for the $\mathrm{CdSe} / \mathrm{CdS}$ nanorods that have three- to fivefold larger volumes. ${ }^{[22]}$ Achievement of ultralow optical gain thresholds under 2PA pumping in the red spectral range, together with our previous demonstrations using green-emitting colloidal nanoplatelets ${ }^{[6]}$ and blue-emitting giant QDs, ${ }^{[14]}$ makes the colloidal semiconductor nanocrystals a highly promising class of materials for frequency up-converted lasers. We calculate the average number of excitons per QD $(\langle N\rangle)$ at the ASE threshold (see Section S2 and S3, Supporting Information) to be 1.43 and 1.24 for 1 PA and 2PA, respectively. These values are in good agreement with the biexciton gain mechanism in our core/shell QD structure, which is similar to type-I QDs.

Recently, colloidal nanoplatelets have emerged as alternative competitors for the optical gain exhibiting low ASE thresholds $\left(\approx 6 \mu \mathrm{J} \mathrm{cm}^{-2}\right)$ and high gain coefficients $\left(650 \mathrm{~cm}^{-1}\right) \cdot{ }^{[6,37,38]}$ Although nanoplatelets will be very advantageous for optical gain, the stability of the ASE in the nanoplatelets is quite limited (after $10^{4}-10^{5}$ laser shots, the ASE intensity drops to half of the initial intensity). ${ }^{[6]}$ Here, we show that our CdSe/CdS QDs exhibit an excellent ASE stability even for laser shots $\approx 1.8 \times 10^{7}$ ( $5 \mathrm{~h}$ of continuous pumping) under a pump intensity of $35 \mu \mathrm{J} \mathrm{cm}^{-2}$ while preserving the initial ASE intensity (a)

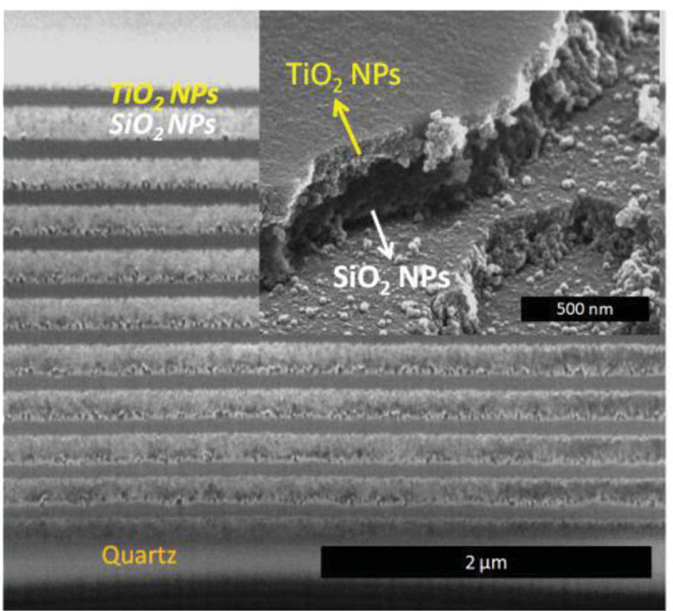

(c)

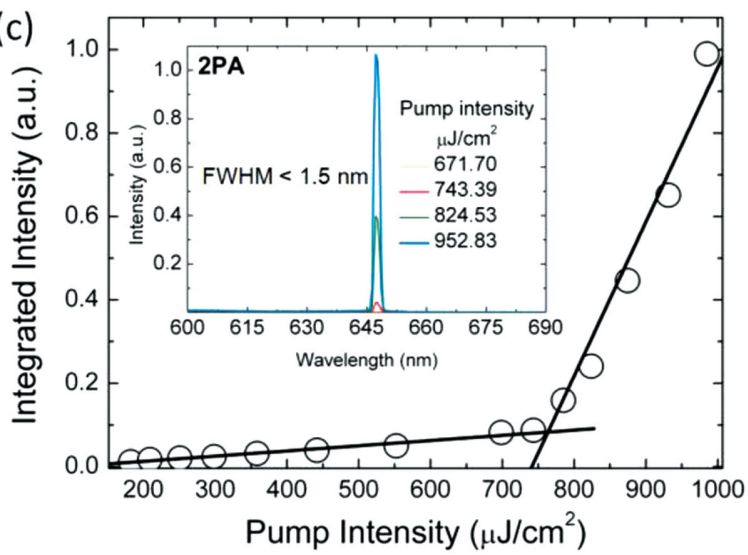

(b)

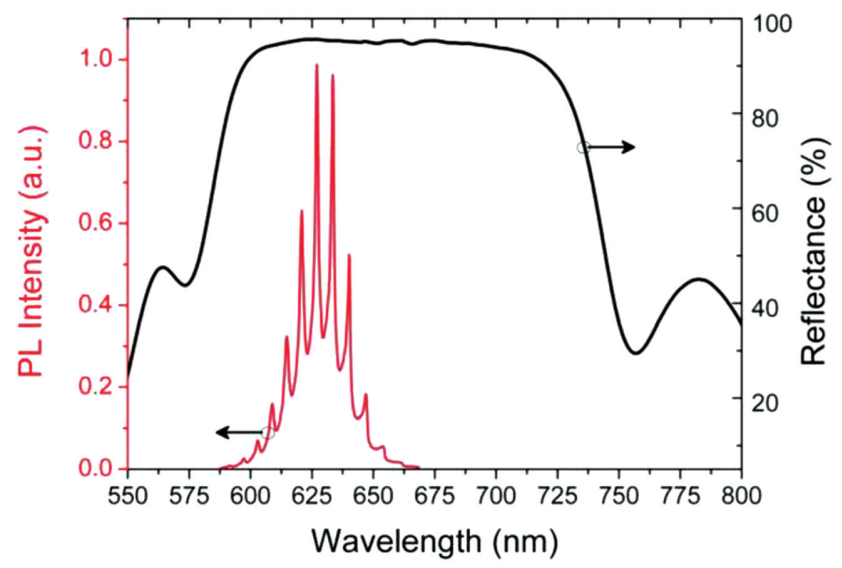

(d)

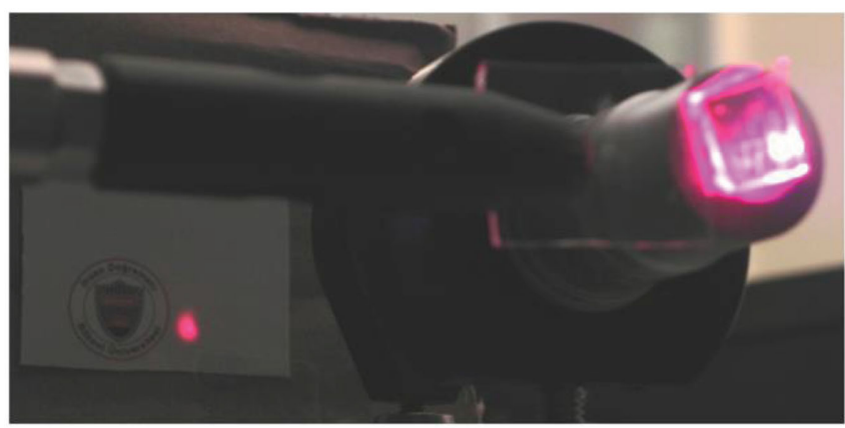

Figure 4. a) Scanning electron microscopy cross-sectional image of the AC DBR shows alternating stacks as the results of different spin-casted nanoparticles $\left(\mathrm{SiO}_{2}\right.$ and $\left.\mathrm{TiO}_{2}\right)$. A focused ion beam was used to create the cross section of the colloidal DBR. The inset shows the close-up of the layered stacks of $\mathrm{TiO}_{2}$ and $\mathrm{SiO}_{2}$ nanoparticles at the cleaved edge of a DBR. b) Surface normal reflectance of the DBR stack with ten-bilayer of TiO ${ }_{2}$ and SiO nanoparticles along with the PL of the QDs in the cavity having optical thickness of about $15 \mu \mathrm{m}$. c) Emission intensity as a function of pump intensity for frequency up-converted AC-VCSEL clearly shows the threshold behavior of the AC-VCSEL. The inset shows the emission spectrum of the laser under different excitation pump densities. Single mode lasing is achieved with a Q-factor greater than 430 . The lasing peak is narrower than 1.5 nm (limited by the spectral resolution of our measurement system). d) Photographic image of the lasing spot from the AC-VCSEL. The spectrally and spatially coherent lasing beam is visible as a bright spot on the screen. 
(see Figure 3c). As shown in Figure 3c, the ASE intensity also exhibits an enhancement in the early time window ${ }^{[22]}$ (up to $1 \times 10^{6}$ laser shots), which is attributed to reaching the thermal equilibrium with partial removal of oxygen and moisture from the solid film sample. Therefore, the stability of our core/shell QDs represents more than two orders of magnitude enhancement over the stability of ASE observed in the nanoplatelets. ${ }^{[6]}$ As compared with other types of colloidal nanocrystals (i.e., nanorods and tetrapods), which have shown stabilities up to $6 \times 10^{6}$ laser shots, ${ }^{[22]}$ our core/shell QDs stand out as an exceptionally stable material for lasers.

With the highly efficient optical gain performance, we used these tailored CdSe/CdS QDs in an all-colloidal laser structure. The most challenging laser configuration — VCSEL — was fully enabled by the most cost-effective technology: solution processing. Two DBRs were made by spin-coating alternating layers of silicon dioxide and titanium dioxide nanoparticles on quartz substrates (see Section S6, Supporting Information for details). ${ }^{[39,40]}$ The densely packed dielectric nanoparticles form multilayered films (Figure 4a) with a considerable refractive index contrast (i.e., $\approx 1.31$ and $\approx 1.95$ for $\mathrm{SiO}_{2}$ and $\mathrm{TiO}_{2}$ layers at $630 \mathrm{~nm}$, respectively) (see Section S8, Supporting Information). The thickness of each layer was controlled to be a quarter of the wavelength at the ASE peak, and the reflectance reaches the maximum of $96 \%$ with ten-bilayers (see Figure $4 \mathrm{~b}$ ). Poly(methyl methacrylate) (PMMA) is then deposited on top of the DBR as the final layer $(\approx 3 \mu \mathrm{m}$ thickness) for protection and creating an optical distance. Then, the core/shell QDs are drop cast on one of the DBRs, and the other DBR was used to sandwich the QD layer so that an all-solution processed and AC-VCSEL was completed (see Figure S5, Supporting Information for the schematic). Epoxy is applied to fix the structure together. PL of the QDs inside the AC-VCSEL is shown in Figure 4b, exhibiting the resonant cavity modes corresponding to an optical thickness of about $15 \mu \mathrm{m}$.

The highly performed QD gain medium with relatively large thickness enabled a frequency up-converted AC-VCSEL with single mode and sharp laser output (Figure 4c, inset) at $647 \mathrm{~nm}$ with a FWHM less than $1.5 \mathrm{~nm}$ (limited by the resolution of the spectrometer). The lasing was observed throughout the whole sample with a small variation of the threshold due to slightly different thickness of the gain medium over the large area of the sample. The average threshold of the ACVCSEL is as low as $764 \mu \mathrm{J} \mathrm{cm}^{-2}$ under 2PA pumping as shown in Figure 4c. This is the lowest threshold for a frequency upconverted laser reported to date even compared to the thresholds achieved by colloidal nanorods and organic semiconductors. ${ }^{[22,41]}$ Depending on the thickness of the drop-cast QD film, multimode lasing in the spectral window of the optical gain medium was also possible, as shown in Figure S6, Supporting Information. Given the relatively low reflectivity of our colloidal DBRs, the ultralow threshold of the VCSEL structure emphasizes the high optical gain capability of our core/shell QDs with the help of the suppressed AR. Unlike alternating polymer-based DBRs, ${ }^{[42]}$ the spectrally and spatially coherent emission of a well-defined laser beam was observed here with a bright spot at the screen behind the sample (Figure 4d). However, the AC-VCSEL did not lase under $400 \mathrm{~nm}$ pumping due to finite absorption and scattering caused by the $\mathrm{TiO}_{2}$ nano- particles (see Figure S7, Supporting Information). This highlights the advantage of 2PA pumping, which avoids unwanted scattering and absorption losses. Frequency up-converted QDbased AC-VCSELs might be promising for sensing and biological applications in the future where the increasing penetration depth and reducing destructive effects of the excitation light are critical.

In conclusion, tailored CdSe-core/CdS-shell QDs are demonstrated as very efficient and highly stable optical gain media thanks to high volume packing density and substantial AR suppression. The ASE performance of the QDs shows very low optical gain thresholds with relatively high gain coefficient, enabling an AC-VCSEL with solution processed DBRs. For the first time, the spatially and spectrally coherent, well-defined laser beam emission from an AC laser is presented especially at a record low threshold in frequency up-converted regime. Our results would certainly extend to other sizes of QDs for different colors, representing a promising future for QD lasers as well as all-solution-processed lasers: the most cost-effective approach for single-material full-color lasers.

\section{Supporting Information}

Supporting Information is available online from the Wiley Online Library or from the author.

\section{Acknowledgements}

The authors would like to thank for the financial support from Singapore National Research Foundation under the programs of NRFRF-2009-09, NRF-CRP-6-2010-02 and the Science and Engineering Research Council, Agency for Science, Technology and Research (A*STAR) of Singapore (Project Nos. 0921010057 and 112120 2009), EU-FP7 Nanophotonics4Energy NoE, and TUBITAK EEEAG 109E002, 109E004, 110E010, 110E217, 112E183, and 114E410. H.V.D. acknowledges support from ESF-EURYI and TUBA-GEBIP. C.D. acknowledges support from NTU's start-up grant. The authors would like to thank Dr. Vijay Kumar Sharma for the help with XPS measurements.

Received: January 27, 2015

Revised: February 24, 2015

Published online: March 24, 2015

[1] S. Nakamura G. Fasol, S. J. Pearton, The Blue Laser Diode: The Complete Story, Springer, Heidelberg, Germany 2000.

[2] C. B. Murray, D. J. Norris, M. G. Bawendi, J. Am. Chem. Soc. 1993, $115,8706$.

[3] C. Dang, J. Lee, C. Breen, J. S. Steckel, S. Coe-Sullivan, A. Nurmikko, Nat. Nanotechnol. 2012, 7, 335.

[4] M. Kazes, D. Y. D. Y. Lewis, Y. Ebenstein, T. Mokari, U. Banin, Adv. Mater. 2002, 14, 317.

[5] C. Grivas, C. Li, P. Andreakou, P. Wang, M. Ding, G. Brambilla, L. Manna, P. Lagoudakis, Nat. Commun. 2013, 4, 2376.

[6] B. Guzelturk, Y. Kelestemur, M. Olutas, S. Delikanli, H. V. Demir, ACS Nano 2014, 8, 6599.

[7] C. Dang, J. Lee, K. Roh, H. Kim, S. Ahn, H. Jeon, C. Breen, J. S. Steckel, S. Coe-Sullivan, A. Nurmikko, Appl. Phys. Lett. 2013, 103, 171104

[8] C. Dang, A. Nurmikko, MRS Bull. 2013, 38, 737. 
[9] I. Moreels, G. Rainò, R. Gomes, Z. Hens, T. Stöferle, R. F. Mahrt, Adv. Mater. 2012, 24, OP231.

[10] V. I. Klimov, A. A. Mikhailovsky, S. Xu, A. Malko, J. A. Hollingsworth, C. A. Leatherdale, H.-J. Eisler, M. G. Bawendi, Science 2000, 290, 314.

[11] Y. Chan, J.-M. Caruge, P. T. Snee, M. G. Bawendi, Appl. Phys. Lett. 2004, 85, 2460.

[12] Y. Kelestemur, A. F. Cihan, B. Guzelturk, H. V. Demir, Nanoscale 2014, 6, 8509.

[13] Y. Wang, V. D. Ta, Y. Gao, T. C. He, R. Chen, E. Mutlugun, H. V. Demir, H. D. Sun, Adv. Mater. 2014, 26, 2954.

[14] B. Guzelturk, Y. Kelestemur, M. Z. Akgul, V. K. Sharma, H. V. Demir, J. Phys. Chem. Lett. 2014, 5, 2214.

[15] V. I. Klimov, A. A. Mikhailovsky, D. W. McBranch, C. A. Leatherdale, M. G. Bawendi, Science 2000, 287, 1011.

[16] I. Robel, R. Gresback, U. Kortshagen, R. Schaller, V. Klimov, Phys. Rev. Lett. 2009, 102, 177404.

[17] M. Zavelani-Rossi, M. G. Lupo, F. Tassone, L. Manna, G. Lanzani, Nano Lett. 2010, 10, 3142.

[18] F. García-Santamaría, Y. Chen, J. Vela, R. D. Schaller, J. A. Hollingsworth, V. I. Klimov, Nano Lett. 2009, 9, 3482.

[19] V. I. Klimov, S. A. Ivanov, J. Nanda, M. Achermann, I. Bezel, J. A. McGuire, A. Piryatinski, Nature 2007, 447, 441.

[20] C. Zhang, F. Zhang, T. Zhu, A. Cheng, J. Xu, Q. Zhang, S. E. Mohney, R. H. Henderson, Y. A. Wang, Opt. Lett. 2008, 33, 2437.

[21] R. Signorini, I. Fortunati, F. Todescato, S. Gardin, R. Bozio, J. J. Jasieniak, A. Martucci, G. Della Giustina, G. Brusatin, M. Guglielmi, D. Giustina, Nanoscale 2011, 3, 4109.

[22] G. Xing, Y. Liao, X. Wu, S. Chakrabortty, X. Liu, E. K. L. Yeow, Y. Chan, T. C. Sum, ACS Nano 2012, 6, 10835.

[23] O. Chen, J. Zhao, V. P. Chauhan, J. Cui, C. Wong, D. K. Harris, H. Wei, H.-S. Han, D. Fukumura, R. K. Jain, M. G. Bawendi, Nat. Mater. 2013, 12, 445.

[24] B. Mahler, N. Lequeux, B. Dubertret, J. Am. Chem. Soc. 2010, 132, 953.
[25] M. Califano, A. Franceschetti, A. Zunger, Nano Lett. 2005, 5, 2360.

[26] F. García-Santamaría, S. Brovelli, R. Viswanatha, J. A. Hollingsworth, H. Htoon, S. A. Crooker, V. I. Klimov, Nano Lett. 2011, 11, 687.

[27] Y.-S. Park, W. K. Bae, L. A. Padilha, J. M. Pietryga, V. I. Klimov, Nano Lett. 2014, 14, 396.

[28] J. Zhao, O. Chen, D. B. Strasfeld, M. G. Bawendi, Nano Lett. 2012, $12,4477$.

[29] X. Wang, X. Ren, K. Kahen, M. A. Hahn, M. Rajeswaran, S. Maccagnano-Zacher, J. Silcox, G. E. Cragg, A. L. Efros, T. D. Krauss, Nature 2009, 459, 686.

[30] G. E. Cragg, A. L. Efros, Nano Lett. 2010, 10, 313.

[31] J. I. Climente, J. L. Movilla, J. Planelles, Small 2012, 8, 754.

[32] D. I. Chepic, A. L. Efros, A. I. Ekimov, M. G. Ivanov, V. A. Kharchenko, I. A. Kudriavtsev, T. V. Yazeva, J. Lumin. 1990, 47, 113.

[33] W. Qin, R. A. Shah, P. Guyot-Sionnest, ACS Nano 2012, 6, 912.

[34] A. F. Cihan, Y. Kelestemur, B. Guzelturk, O. Yerli, U. Kurum, H. G. Yaglioglu, A. Elmali, H. V. Demir, J. Phys. Chem. Lett. 2013, 4, 4146.

[35] Y. Liao, G. Xing, N. Mishra, T. C. Sum, Y. Chan, Adv. Mater. 2012, 24, 159.

[36] J. J. J. Jasieniak, I. Fortunati, S. Gardin, R. Signorini, R. Bozio, A. Martucci, P. Mulvaney, Adv. Mater. 2008, 20, 69.

[37] C. She, I. Fedin, D. S. Dolzhnikov, A. Demortière, R. D. Schaller, M. Pelton, D. V Talapin, D. Richard, Nano Lett. 2014, 14, 2772.

[38] J. Q. Grim, S. Christodoulou, F. Di Stasio, R. Krahne, R. Cingolani, L. Manna, I. Moreels, Nat. Nanotechnol. 2014, 9, 891.

[39] D. P. Puzzo, L. D. Bonifacio, J. Oreopoulos, C. M. Yip, I. Manners, G. A. Ozin, J. Mater. Chem. 2009, 19, 3500.

[40] S. Colodrero, M. Ocaña, H. Míguez, Langmuir 2008, 24, 4430.

[41] C. Bauer, B. Schnabel, E.-B. Kley, U. Scherf, H. Giessen, R. F. Mahrt, Adv. Mater. 2002, 14, 673

[42] V. M. Menon, M. Luberto, N. V Valappil, S. Chatterjee, Opt. Express 2008, 16, 19535. 